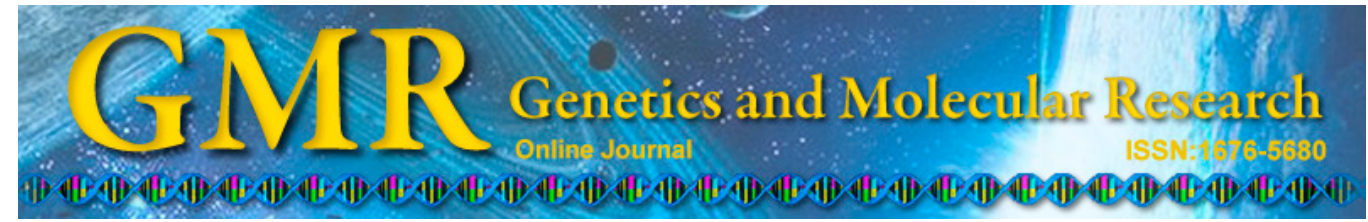

\title{
Benefits of minimizing immunosuppressive dosage according to cytochrome P450 3 A5 genotype in liver transplant patients: findings from a single-center study
}

L. Wang ${ }^{1}$, N. Li ${ }^{2}$, M.X. Wang ${ }^{3}$ and S.C. Lu$^{4}$

${ }^{1}$ Liver Transplant Center, Beijing You An Hospital, Capital Medical University, Beijing, China

${ }^{2}$ President's Office, Beijing You An Hospital, Capital Medical University, Beijing, China

${ }^{3}$ Pharmacology Base, Beijing You An Hospital, Capital Medical University, Beijing, China

${ }^{4}$ Department of Hepatobiliary Surgery, General Hospital of PLA, Beijing, China

Corresponding author: S.C. Lu

E-mail: lushichun201449@163.com

Genet. Mol. Res. 14 (2): 3191-3199 (2015)

Received May 14, 2014

Accepted October 27, 2014

Published April 10, 2015

DOI http://dx.doi.org/10.4238/2015.April.10.31

ABSTRACT. We evaluated the clinical efficacy of tailoring tacrolimus
dosage to cytochrome P450 (CYP) 3A5 genotype in liver transplant
patients. One hundred patients who received tacrolimus-based therapy
were included in the retrospective study in which the relationship between
the tacrolimus blood trough concentration/dosage ratio and the CYP3A5
genotype of both donors and recipients was determined. Subsequently, 106
patients were continuously enrolled in a prospective study and followed-
up for 6 months; the relationship between tacrolimus dosage and CYP3A5
genotype was also determined. Rates of acuterejection, hepatotoxicity, renal
toxicity, neurotoxicity, hypertension, and hyperglycemia were compared
between the groups. During the 6 months following liver transplantation,
the mean tacrolimus concentration/dosage ratio among patients who did
not have the CYP3A5*1 genotype and who received a transplant from a 
donor with the same genotype $(24 / 100,24 \%$ of patients) was higher than that among patients who did have the CYP $3 \mathrm{~A} 5 * 1$ genotype and/or had a donor with the same genotype (76/100, $76 \%$ of patients). In the second part of the study, the tacrolimus dosage was tailored to CYP3A5 genotype and 24 patients $(22.64 \%)$ received a lower dose. There was an obvious decrease in acute rejection, hepatotoxicity, renal toxicity, neurotoxicity, hypertension, hyperglycemia, and Pneumocystis carinii infection among the latter group. A lower tacrolimus dose was suitable for about $25 \%$ of the liver transplant patients, as these patients did not have the CYP3A $5 * 1$ genotype and received a transplant from a donor with the same genotype. Tailoring the tacrolimus dosage according to the CYP3A5 genotype could reduce rejection and adverse effects.

Key words: Cytochrome P450 3A5; Liver transplantation; Tacrolimus; Transplant rejection; Renal toxicity

\section{INTRODUCTION}

Research in cases of renal, lung, and heart transplantation has confirmed that there is a strong correlation between cytochrome $P 450$ (CYP) $3 A 5$ gene polymorphism and patient-specific differences in responses to tacrolimus (Zheng et al., 2003, 2004; Macphee et al., 2005; Li et al., 2007; Zuo et al., 2013). However, the situation is more complex in cases of liver transplantation. The CYP3A5 enzyme is distributed in both the liver and the small intestine, and the genetic backgrounds of both the donor and the recipient affect tacrolimus metabolism. It is not clear which has the greatest influence. Therefore, we performed a retrospective analysis to explore the major factors affecting the tacrolimus blood trough concentration. Subsequently, we conducted a prospective study in which we tailored the tacrolimus dosage to the patient's CYP3A5 genotype and observed the clinical effects.

\section{MATERIAL AND METHODS}

\section{Retrospective study}

The retrospective study included 100 adult Chinese orthotopic liver transplantation patients who were treated with tacrolimus at our center between January 2010 and December 2011. Tacrolimus was administered orally twice daily (at 6:00 and 18:00 h). The average initial dose was 4-6 mg/day, but this was adjusted based on the physician's judgment, clinical syndromes, trough concentration, renal function, and immune function. In addition, we determined the CYP $3 A 5$ gene polymorphism status of both donors and recipients and calculated the blood trough concentration/dosage $(\mathrm{C} / \mathrm{D})$ ratio of tacrolimus for the 6 months after transplantation. Subsequently, we analyzed the relationship between the C/D ratio of tacrolimus and the CYP3A5 genotypes of donors and recipients. Concentration values influenced by liver dysfunction, intestine dysfunction, fluconazole therapy, amlodipine, nicardipine, or a lack of regular outpatient visits were excluded. 


\section{Prospective study}

In 2012, we continuously enrolled 106 adult orthotopic liver transplantation patients in a prospective study. Their CYP3A5 genotypes were determined at the time of transplantation and tacrolimus-based immunosuppressive therapy was tailored to each patient's genetic profile. Among CYP3A $5 * 1 * 1 / * 1 * 3$ or donor CYP3A $5 * 1 * 1 / * 1 * 3$ patients, the initial dose of tacrolimus was $4 \mathrm{mg} /$ day, which was soon increased to $6 \mathrm{mg} /$ day. Among donors and recipients who both had the CYP3A $5 * 3 * 3$ genotype, the initial dose of tacrolimus was $2 \mathrm{mg} /$ day and this was increased to $3-4 \mathrm{mg} /$ day. We took great care when increasing the tacrolimus dosage in CYP $3 A 5 * 3 * 3$ patients who also had a CYP3A $5 * 3 * 3$ donor. The incidence of acute rejection, hepatotoxicity, renal toxicity, neurotoxicity, hypertension, and hyperglycemia was compared among the 100 patients treated in 2010 and 2011, and the 106 patients treated in 2012. The study was approved by the hospital Ethics Committee [approval number: 2013 (8)].

Diagnoses of acute rejection and hepatotoxicity were dependent on clinical symptoms, liver function, and liver biopsy findings. The following definitions were used for each condition:

Liver toxicity: glutamic-pyruvic transaminase levels exceed the upper limit and/or bilirubin levels exceed $50 \%$ of the upper limit of normal.

Diagnosis of renal toxicity: preoperative serum creatinine $>167 \mu \mathrm{M}$ in patients with normal renal function or show a $>30 \%$ increase postoperatively in patients with abnormal renal function before surgery (after the exclusion of other factors).

Diagnosis of neurotoxicity: hand tremor, numb lips, and numbness in limbs.

Hypertension: systolic pressure $>140 \mathrm{mmHg}(1 \mathrm{mmHg}=0.133 \mathrm{kPa})$ or diastolic pressure $>90 \mathrm{mmHg}$.

Hyperglycemia: blood glucose $>7.0 \mathrm{mM}$ for three consecutive days.

\section{Genotype analysis}

Peripheral blood samples or liver specimens were obtained from recipients and donors and DNA was extracted. The single nucleotide polymorphism A6986G in CYP3A5 intron 3 was identified by polymerase chain reaction-restriction fragment length polymorphism.

The tacrolimus concentration $(\mathrm{ng} / \mathrm{mL})$ was identified by microparticle enzyme immunoassay using an IMx analyzer (Abbott Japan, Tokyo, Japan). In total, 1854 blood samples were collected and the daily dosage of tacrolimus was recorded at the detection spot.

\section{Statistical analysis}

All concentration values are reported as means $\pm \mathrm{SD}$. The distribution of genotypes was analyzed using the chi-squared test. A non-parametric test was used to compare differences in $\mathrm{C} / \mathrm{D}$ ratios of tacrolimus among groups. All statistical analyses were performed using the SPSS v.17.0 software. A P value $<0.05$ was considered to be statistically significant.

\section{RESULTS}

\section{Patients}

In total, 206 liver transplantation patients were included in both studies; there were no 
instances of living donor transplantation. The demographic characteristics of the 206 patients are shown in Table 1. The mean age of the liver transplant recipients was $49.06 \pm 8.64$ years.

Table 1. Characteristics of the study population $(\mathrm{N}=206)$.
\begin{tabular}{lcc}
\hline Demographics & Patients treated in 2010-2011 (N=100) & Patients treated in 2012 (N = 106) \\
\hline Gender (male/female) & $78 / 22$ & $81 / 25$ \\
Age (years) & $49.58 \pm 9.48$ & $48.578 \pm 7.77$ \\
Primary disease & 44 & 55 \\
Cirrhosis & 38 & 39 \\
Cancer & 5 & 6 \\
Primary biliary cirrhosis & 2 & 4 \\
Alcoholic cirrhosis & 1 & 2 \\
Other (drug-induced liver injury) & & \\
\hline
\end{tabular}

\section{Genotype data}

The CYP3A5 genotype frequencies in donors and recipients are summarized in Table 2. There was no statistically significant difference between recipients and donors in terms of the proportion of patients with each genotype $(\mathrm{P}>0.05)$. In approximately $25 \%$ of the liver transplant patients, neither the donor nor the recipient had the CYP3A $5 * 1$ genotype.

Table 2. Pharmacogenomic data for the study population $(\mathrm{N}=206)$.

\begin{tabular}{lcc}
\hline Genotype frequency & Patients treated in $2010-2011(\mathrm{~N}=100)$ & Patients treated in $2012(\mathrm{~N}=106)$ \\
\hline Donor $* 1 / * 1$ and $* 1 / * 3+$ recipient $* 1 / * 1$ and $* 1 / * 3$ & $31(31 \%)$ & $20(18.87 \%)$ \\
Donor $* 1 / * 1$ and $* 1 / * 3+$ recipient $* 3 / * 3$ & $21(21 \%)$ & $26(24.53 \%)$ \\
Donor $* 3 / * 3+$ recipient $* 1 / * 1$ and $* 1 / * 3$ & $24(24 \%)$ & $35(33.02 \%)$ \\
Donor $* 3 / * 3+$ recipient $* 3 / * 3$ & $24(24 \%)$ & $25(23.58 \%)$ \\
\hline
\end{tabular}

\section{Influence of pharmacogenomic data on tacrolimus $\mathrm{C} / \mathrm{D}$ ratio}

To investigate the effects of CYP $3 A 5$ gene polymorphisms in donors and recipients on tacrolimus C/D ratio, we divided the patients who were treated in 2010-2011 $(\mathrm{N}=100)$ into four groups according to patient and donor genotype and then compared the tacrolimus C/D ratios among the groups. Because patient-specific with one CYP3A5*1 allele express CYP3A5 (Kuehl et al., 2001), the CYP3A5*1/*1 and CYP3A5*1/*3 genotypes were combined and defined as "expressed"; the CYP 3 A $5 * 3 / * 3$ genotype was defined as "not expressed". Table 3 shows the tacrolimus C/D ratios for the four groups. Age, gender, and weight were not significantly different between the patients included in the retrospective and prospective studies.

As shown in Table 3, during the 6-month postoperative follow-up period, patients with the CYP $3 \mathrm{~A} 5 * 3 / * 3$ genotype whose donors had the same genotype had higher tacrolimus $\mathrm{C} / \mathrm{D}$ ratios than patients in the other three groups. In addition, the tacrolimus $\mathrm{C} / \mathrm{D}$ ratio of patients who had the same CYP3A5*1 genotype as their donor was lower than that in the other three groups, but the difference was not significant. To further investigate this hypothesis, we expanded our dataset by analyzing all 206 patients enrolled during 2010-2012, and the outcome was the same (Figure 1). 
Table 3. Concentration/dosage ratios $(\mathrm{ng} / \mathrm{mL}) /\left(\mathrm{mg} \cdot \mathrm{kg}^{-1} \cdot \mathrm{day}^{-1}\right)$ of tacrolimus 6 months after transplantation among patients treated in 2010-2011 and grouped according to donor and recipient cytochrome P450 3A5 genotype $(\mathrm{N}=100)$.

\begin{tabular}{lcccc}
\hline $\begin{array}{l}\text { Donor } \\
\text { Recipient }\end{array}$ & $\begin{array}{c}* 1 / * 1 \text { and } * 1 / * 3 \\
* 1 / * 1 \text { and } * 1 / * 3\end{array}$ & $\begin{array}{c}* 1 /{ }_{1} \text { and } * 1 / * 3 \\
* 3 / * 3\end{array}$ & $\begin{array}{c}* 3 / * 3 \\
* 1 / * 1 \text { and } * 1 / * 3\end{array}$ & $\begin{array}{c}* 3 / * 3 \\
* 3 / * 3\end{array}$ \\
\hline Number & 31 & 21 & 24 & 24 \\
Week 1 & $44.06 \pm 49.50$ & $55.86 \pm 53.95$ & $49.82 \pm 41.41$ & $137.55 \pm 123.96 *$ \\
Week 2 & $41.10 \pm 48.12$ & $59.94 \pm 61.20$ & $53.36 \pm 48.40$ & $121.84 \pm 109.26 *$ \\
Week 3 & $35.46 \pm 31.50$ & $56.51 \pm 52.50$ & $50.65 \pm 48.40$ & $134.28 \pm 129.28 *$ \\
Week 4 & $48.55 \pm 46.10$ & $61.86 \pm 56.87$ & $57.20 \pm 57.15$ & $125.54 \pm 121.73 *$ \\
Month 2 & $60.38 \pm 61.05$ & $79.00 \pm 75.60$ & $79.02 \pm 79.13$ & $155.83 \pm 156.96 *$ \\
Month 3 & $67.09 \pm 66.20$ & $60.09 \pm 58.12$ & $61.11 \pm 58.50$ & $133.98 \pm 136.00 *$ \\
Month 4 & $51.44 \pm 50.15$ & $65.38 \pm 63.55$ & $62.83 \pm 61.85$ & $144.79 \pm 134.75 *$ \\
Month 5 & $57.27 \pm 59.40$ & $82.30 \pm 78.53$ & $64.43 \pm 63.90$ & $133.41 \pm 122.80 *$ \\
Month 6 & $72.89 \pm 72.33$ & $89.80 \pm 83.82$ & $201.66 \pm 197.06 *$ \\
\hline
\end{tabular}

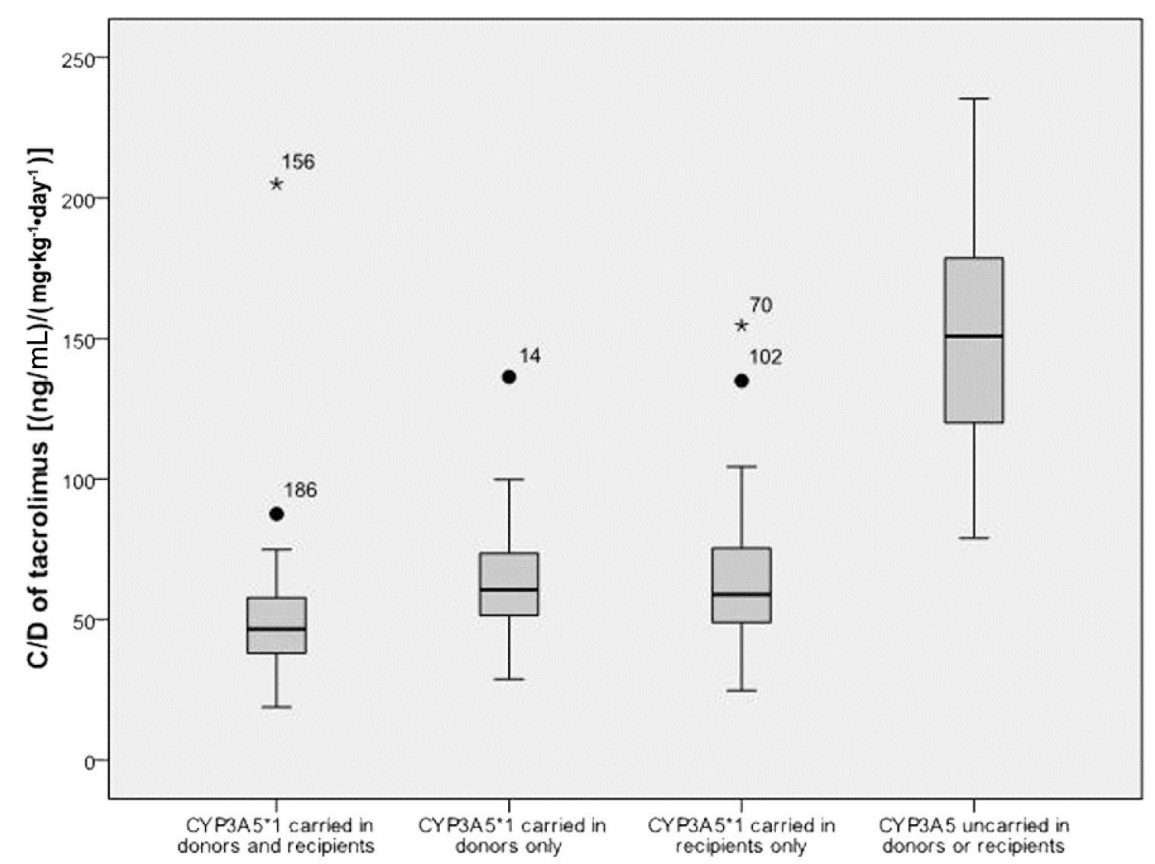

Figure 1. Tacrolimus concentration/dosage ratio among the four groups classified according to combination donor and recipient cytochrome P4503A5 genotypes $(\mathrm{N}=206)$.

\section{Effect of tailoring tacrolimus dosage according to CYP3A5 genotype}

We divided the 206 patients into two groups. Group $1(\mathrm{~N}=100)$ comprised patients who were treated in 2010-2011 and whose tacrolimus dosage was adjusted in accordance with the judgment of the physician. Group $2(\mathrm{~N}=106)$ comprised the patients treated in 2012 ; their tacrolimus dosage was adjusted according to their CYP3A5 genotype. The average daily dose of tacrolimus and the rates of acute rejection and other complications were then compared between the two groups (Tables 4 and 5). 
Table 4. Average dosage of tacrolimus between the two groups of patients during 6-month follow-up after transplantation.

\begin{tabular}{lc}
\hline Group & Average dosage $(\mathrm{mg} /$ day $)$ \\
\hline Patients treated in $2010-2011(\mathrm{~N}=100)$ & $42.94 \pm 6.29$ \\
Patients treated in $2012(\mathrm{~N}=106)$ & $45.76 \pm 1.42$ \\
Donor recipient $* 1 / * 1$ and $* 1 * 3(\mathrm{~N}=82)$ & $31.5 \pm 2.32$ \\
Donor $* 3 / * 3+$ recipient $* 3 / 3(\mathrm{~N}=24)$ & \\
\hline
\end{tabular}

Table 5. Rates of acute rejection and of complications in the two patient groups during the 6-month follow-up after transplantation.

\begin{tabular}{lcc}
\hline & Patients treated in 2010-2011 $(\mathrm{N}=100)$ & Patients treated in 2012 $(\mathrm{N}=106)$ \\
\hline Acute rejection & $19 / 100(19 \%)$ & $12 / 106(11.32 \%)$ \\
Hepatotoxicity & $9 / 100(9 \%)$ & $6 / 106(5.66 \%)$ \\
Renal toxicity & $25 / 100(25 \%)$ & $13 / 106(12.26 \%)$ \\
Neurotoxicity & $11 / 100(11 \%)$ & $6 / 106(5.66 \%)$ \\
Hypertension & $24 / 100(24 \%)$ & $8 / 106(7.55 \%)$ \\
Hyperglycemia & $24 / 100(24 \%)$ & $18 / 106(16.98 \%)$ \\
Pneumocystis carinii pneumonia infection & $4 / 100(4 \%)$ & $1 / 106(1.89 \%)$ \\
\hline
\end{tabular}

\section{DISCUSSION}

Tacrolimus is a widely used immunosuppressant; it has a narrow therapeutic index and response varies greatly among patients, which makes setting a suitable dosage for transplant patients difficult. Apart from monitoring blood concentration, T lymphocyte function, liver function fluctuations, liver biopsy, and pharmacokinetic genetics can be used to adjust the dosage. Tacrolimus is metabolized by both CYP3A4 and CYP3A5, but CYP3A5 plays a more important role. CYP $3 A 5$ gene polymorphism influences the $\mathrm{C} / \mathrm{D}$ ratio of tacrolimus (López-Montenegro Soria et al., 2010); an A to G sequence variant at the 6986 nucleotide in CYP3A5 gene intron 3 causes loss of functional CYP3A5. Studies of lung, heart, and renal transplantation have confirmed that CYP3A5*1 (6986A) plays an important role in tacrolimus metabolism (Chen et al., 2009).

However, in liver transplantation, the contributions of the donor liver and recipient gut complicate tacrolimus metabolism. The relationship between CYP3A5 polymorphism and variations in patient-specific responses to tacrolimus has rarely been studied in liver transplant patients, and studies that have been performed have reported contradictory findings.

One study of 53 liver transplantation patients by Yu et al. (2006) suggested that the donor CYP $3 \mathrm{~A} 5 * 3$ genotype made a greater contribution to the large inter-patient-specific variation in tacrolimus dose requirement than gut CYP3A5. Goto et al. (2004) indicated that the pharmacokinetics of tacrolimus are influenced by the graft CYP3A5 genotype. Yet another study revealed that patients with a liver graft with the CYP3A5*1 allele had a lower C/D ratio of tacrolimus than patients with a liver graft with the CYP $3 \mathrm{~A} 5 * 3 / * 3$ genotype (Rahsaz et al., 2012). Other studies have highlighted the contribution of the recipient's CYP3A5 genotype to tacrolimus metabolism. Shi et al. (2013) studied tacrolimus pharmacokinetics and concluded that patient-specific differences in tacrolimus response could probably be explained by different intestinal CYP3A5 genotypes. A significant difference in tacrolimus daily dose and C/D ratios has also been reported between recipients with the CYP3A5 6986GG allele and 
those with the CYP3A5 6986AG/AA allele within 6 months of transplantation (Provenzani et al., 2011). A previous study also found that the genetic polymorphism of intestinal CYP3A5 in liver transplant patients was an important factor affecting tacrolimus blood concentration (Uesugi et al., 2006).

In our study, we divided the patients into four groups according to donor and recipient genotype, and we compared the tacrolimus C/D ratio among these groups to investigate the relationship between CYP3A5 polymorphisms and tacrolimus metabolism. Concentration values influenced by fluconazole and nicardipine (Hooper et al., 2012) were excluded. We found that the tacrolimus C/D ratios of patients with the CYP $3 \mathrm{~A} 5 * 3 / * 3$ genotype who received a transplant from a donor who had the same genotype were greater than those of the other three groups, while no significant difference was found among the other three groups themselves. This finding confirms that both the hepatic and intestinal CYP3A5*1 alleles significantly influence the tacrolimus dose and C/D ratio. In other words, only patients who had the CYP3A $5 * 3 * 3$ genotype and whose donor had the same genotype had higher tacrolimus C/D ratios within the 6 months after the operation and required a lower tacrolimus dose to achieve immune tolerance. This finding is partly supported by other research (Elmachad et al., 2012; García-Roca et al., 2012), and also enabled us to tailor the immunosuppressant dose according to CYP3A5 genotype. Based on the findings from the retrospective study, we tailored the tacrolimus dosage in the prospective study to match the CYP3A5 genotypes of the donor and recipient. Among patients with the CYP $3 \mathrm{~A} 5 * 3 * 3$ genotype whose donor had the same genotype, the tacrolimus dosage was decreased to $2 \mathrm{mg}$ /day initially and then slowly increased to 3-4 $\mathrm{mg} /$ day; these doses are lower than those prescribed for patients and/or donors with the CYP3A $5 * 1$ genotype.

After 6 months of follow-up, the total dosage of tacrolimus in patients with the CYP3A $5 * 3 * 3$ genotype who had a donor with that genotype was lower than that among patients in the other groups; no difference in the $\mathrm{C} / \mathrm{D}$ ratio of tacrolimus was detected. Safety comparisons of the 106 patients in the prospective study and the 100 patients in the retrospective study indicated that the rates of acute rejection, hepatotoxicity, renal toxicity, neurotoxicity, hypertension, hyperglycemia, and $P$. carinii pneumonia infection were all lower among patients in the prospective study. It is not clear whether this protocol is suitable for every patient owing to the short follow-up period, but we inferred that approximately $25 \%$ of patients had the same CYP3A $5 * 3 / * 3$ genotype as their donor, required a reduced dosage of tacrolimus, and benefitted from this during the 6 months after transplantation.

However, the rates of acute rejection and adverse effects were not greatly improved, especially the rates of hyperglycemia and hypertension. In addition, we discovered that some patients were unsuited to tacrolimus therapy; their clinical symptoms included intractable elevated bilirubin levels or refractory hyperglycemia. It is worth mentioning that all of these individuals were CYP $3 \mathrm{~A} 5 * 1 * 1 / * 1 * 3$ patients or CYP $3 \mathrm{~A} 5 * 1 * 1 / * 1 * 3$ donors, and that immunosuppressant therapy was switched from tacrolimus to cyclosporine A for some of them.

Tacrolimus is not suitable for patients whose donors have identical CYP3A $5 * 1 * 1 / * 1 * 3$ genotypes because other enzymes also contribute to the metabolism of tacrolimus, including MDR1 and CYP3A4. For these patients, switching the immunosuppressant is more effective. Further research is required to improve the protocol for tailoring immunosuppressant therapy to CYP3A5 genotype.

In conclusion, our findings suggest that both donor and recipient CYP3A5 polymorphisms affect the C/D ratio of tacrolimus. Approximately $25 \%$ of patients had the 
same CYP3A $5 * 3 * 3$ genotype as their donors and benefitted from a reduction in the tacrolimus dose. The reduced incidence of side effects proved that it is reasonable to tailor the tacrolimus dose to match the genotypes of donors and recipients, but further research is required to assess the long-term benefits.

\section{Conflicts of interest}

The authors declare no conflict of interest.

\section{ACKNOWLEDGMENTS}

Research supported by the National Science and Technology Fund (Grant\#30671977), the Capital Development Fund of Medicine (Grant \#2005-2034), the Capital Health Development Special Funds (Grant \#2011-2018-03), the Beijing Municipal Health Bureau (Grant \#2011-2-18), and the Organ Transplantation Research Fund from the Ministry of Health (Grant \#RHECC08-2012-08).

\section{REFERENCES}

Chen B, Zhang W, Fang J, Jin Z, et al. (2009). Influence of the MDR1 haplotype and CYP3A5 genotypes on cyclosporine blood level in Chinese renal transplant recipients. Xenobiotica 39: 931-938.

Elmachad M, Elkabbaj D, Elkerch F, Laarabi FZ, et al. (2012). Frequencies of CYP3A5*1/*3 variants in a Moroccan population and effect on tacrolimus daily dose requirements in renal transplant patients. Genet. Test Mol. Biomarkers 16: 644-647.

García-Roca P, Medeiros M, Reyes H, Rodríguez-Espino BA, et al. (2012). CYP3A5 polymorphism in Mexican renal transplant recipients and its association with tacrolimus dosing. Arch. Med. Res. 43: 283-287.

Goto M, Masuda S, Kiuchi T, Ogura Y, et al. (2004). CYP3A5*1-carrying graft liver reduces the concentration/oral dose ratio of tacrolimus in recipients of living-donor liver transplantation. Pharmacogenetics 14: 471-478.

Hooper DK, Fukuda T, Gardiner R, Logan B, et al. (2012). Risk of tacrolimus toxicity in CYP3A5 nonexpressors treated with intravenous nicardipine after kidney transplantation. Transplantation 93: 806-812.

Kuehl P, Zhang J, Lin Y, Lamba J, et al. (2001). Sequence diversity in CYP3A promoters and characterization of the genetic basis of polymorphic CYP3A5 expression. Nat. Genet. 27: 383-391.

Li D, Lu W, Zhu JY, Gao J, et al. (2007). Population pharmacokinetics of tacrolimus and CYP3A5, MDR1 and IL-10 polymorphisms in adult liver transplant patients. J. Clin. Pharm. Ther. 32: 505-515.

López-Montenegro Soria MA, Kanter Berga J, Beltrán Catalán S, Milara Payá J, et al. (2010). Genetic polymorphisms and individualized tacrolimus dosing. Transplant Proc. 42: 3031-3033.

Macphee IA, Fredericks S, Mohamed M, Moreton M, et al. (2005). Tacrolimus pharmacogenetics: the CYP3A5*1 allele predicts low dose-normalized tacrolimus blood concentrations in whites and South Asians. Transplantation 79: 499-502.

Provenzani A, Notarbartolo M, Labbozzetta M, Poma P, et al. (2011). Influence of CYP3A5 and ABCB1 gene polymorphisms and other factors on tacrolimus dosing in Caucasian liver and kidney transplant patients. Int. J. Mol. Med. 28: 1093-1102.

Rahsaz M, Azarpira N, Nikeghbalian S, Aghdaie MH, et al. (2012). Association between tacrolimus concentration and genetic polymorphisms of CYP3A5 and ABCB1 during the early stage after liver transplant in an Iranian population. Exp. Clin. Transplant. 10: 24-29.

Shi Y, Li Y, Tang J, Zhang J, et al. (2013). Influence of CYP3A4, CYP3A5 and MDR-1 polymorphisms on tacrolimus pharmacokinetics and early renal dysfunction in liver transplant recipients. Gene 512: 226-231.

Uesugi M, Masuda S, Katsura T, Oike F, et al. (2006). Effect of intestinal CYP3A5 on postoperative tacrolimus trough levels in living-donor liver transplant recipients. Pharmacogenet. Genomics 16: 119-127.

Yu SF, Wu LH and Zheng SS. (2006). Genetic factors for individual administration of immunosuppressants in organ transplantation. Hepatobiliary Pancreat. Dis. Int. 5: 337-344.

Zheng H, Webber S, Zeevi A, Schuetz E, et al. (2003). Tacrolimus dosing in pediatric heart transplant patients is related to CYP3A5 and MDR1 gene polymorphisms. Am. J. Transplant 3: 477-483. 
Zheng H, Zeevi A, Schuetz E, Lamba J, et al. (2004). Tacrolimus dosing in adult lung transplant patients is related to cytochrome P4503A5 gene polymorphism. J. Clin. Pharmacol. 44: 135-140.

Zuo XC, Ng CM, Barrett JS, Luo AJ, et al. (2013). Effects of CYP3A4 and CYP3A5 polymorphisms on tacrolimus pharmacokinetics in Chinese adult renal transplant recipients: a population pharmacokinetic analysis. Pharmacogenet. Genomics 23: 251-261. 